\title{
Mejoría de la gravedad de la adicción en los tratamientos con metadona*
}

\author{
*vimeno C ; ***Esteban J; ***Pellin MC, *** Climent J.M \\ Y Grupo de Investigación en Adicciones de Alicante: * ${ }^{\circ}$ Camacho I; *'Gimeno M.J;

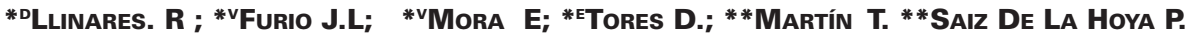 \\ *Unidad de Conductas Adictivas ${ }^{* D}$ Denia, ${ }^{* E}$ Elche, ${ }^{*}$ La Vila Joiosa **Penitenciario Fontcalen \\ *** Universidad Miguel Hernández. *** Hospital General Alicante. \\ Enviar correspondencia: \\ Carmen Gimeno Escrig. Unidad Conductas Adictivas. C/ Hernan Cortes n 9. 03570 La Vila Joiosa. Alacant \\ TF/Fax: 966850953 /966 851 207. E mail: vilauca@terra.es
}

\section{RESUMEN}

Introducción: Los resultados de los tratamientos con metadona se han evaluado mediante la consecución de la abstinencia de sustancias, la retención, la disminución de los delitos, y la integración socio-laboral. Esta perspectiva tradicional se ha ampliado con la reducción de daños y con instrumentos de medición que integran todos estos aspectos.

Objetivo: Evaluar la gravedad de la adicción y valorar los resultados al año de seguimiento.

Diseño: Estudio prospectivo, longitudinal y multicéntrico.

Pacientes y método: 197 pacientes en tratamiento de mantenimiento con metadona. Medición con el Indicador del Tratamiento de la Adicción a Opiáceos utilizado al mes y al año de tratamiento.

Resultados: La retención al año fue del 57\%. El 3\% continuaban consumiendo diariamente heroína, y un $9 \%$ cocaína. La dosis media de los pacientes retenidos fue $56 \mathrm{mg} / \mathrm{dí}$. No hubo diferencias en las dosis iniciales de los pacientes que abandonaron y los que continuaron el tratamiento Disminuyó el hábito de compartir jeringuillas desde el 13\% al inicio hasta el $8 \%$ al año. La gravedad de la adicción mostró mejoría significativa entre la valoración inicial y la final. Aquellos pacientes que habían cometido delitos tenían un riesgo 2,8 veces superior de dejar el tratamiento que los que carecían de antecedentes. El $89 \%$ de los pacientes valoraron el tratamiento como muy bueno o bueno.

Discusión: Existe una mejoría en el funcionamiento global, aunque persisten las carencias en la esfera social y de ajuste psicológico. La existencia de delitos predice el abandono.

Palabras clave: Programa mantenimiento con metadona, evaluación, tratamiento, Indicador de Tratamiento con Opiáceos

\section{SUMMARY}

Introduction: results from methadone treatments have been assessed according to the following criteria: abstinence, retention, delinquency-rate reduction, social and labour integration. This traditional view has been extended to include harm-reduction. Furthermore, we have employed measuring instruments covering all the above mentioned aspects.

Objetive: to assess addiction severity as well as to study the results of the one-year follow-up period.

Design: a prospective, longitudinal and multi-centre study.

Patients and method: 197 patients under methadone treatment. The measuring instrument employed was the Opiate Treatment Index (OTI). It was used one month and one year after treatment intake.

Results: After one year of undergoing treatment, retention rates amounted to $57 \%$. $3 \%$ of the sample was still consuming heroin daily, $9 \%$ was consuming cocaine. The average dose for retained patients was $56 \mathrm{mg} /$ day. Both dropouts and retained patients were administered the same initial doses. Needle-sharing habits decreased from an initial $13 \%$ to $8 \%$ at the conclusion of the year long period. Addiction severity was significantly lower at the final assessment. Patients with a delinquency record were 2.8 times more likely to drop out than patients without one. $89 \%$ of patients considered the treatment to be good or very good.

Discussion: An improvement is observed at a global operating level. However, both social and psychological adjustment deficiencies remain. Delinquency is usually a dropout predictor.

Key words: methadone maintenance, assessment, Opiate Treatment Index (OTI).

*Financiado por el Instituto de Investigación en Drogodependencias (INID), Universidad Miguel Hernández. 


\section{INTRODUCCIÓN}

$\mathbf{L}$ os primeros tratamientos con metadona fueron desarrollados en Canadá durante los años 50 por Hallyday y Dole y Nyswander en la década de los 60 en Estados Unidos (Verstee; 2000). Surgieron como un programa alternativo al objetivo de abstinencia que hasta entonces había constituido la única posibilidad razonable para los dependientes de opiáceos.

Los Tratamientos de Mantenimiento con Metadona (TMM) ayudan a proporcionar una asistencia al drogodependiente tanto en los cuidados de salud como en la situación social. Favorecen una disminución en el consumo de drogas por vía parenteral, una disminución de los daños derivados de la transmisión de enfermedades infecciosas como el sida o las hepatitis y una disminución en la actividad delictiva relacionada con las drogas (Baker, et al. 1993; Baker, et al. 1995; Mino, 1995; Duro, 1995; O Hare, 1995; Murray, 1998; Weber, 1998; Hartel, 1998; Des Jarlais, 1998; Marsch, 1998; Ward, et al. 1999, Bell et al 2000, Fernández, 1999, Sánchez 2000, Fernández 2001).

Para cumplir con estos objetivos, los TMM han de facilitar el acceso del mayor número de pacientes posibles, ampliando los criterios de inclusión, utilizando las dosis adecuadas y sin límites en el tiempo de tratamiento (De La Fuente 1996; Lowinston, et al. 1997; D'Ippoliti, et al. 1998), así como restringiendo los criterios de exclusión y eliminando al máximo los tramites administrativos.

Para la evaluación clásica de los programas de mantenimiento con metadona se han destacado cuatro actuaciones: en primer lugar, la retención (Kreek, 1983; Gaughwin, 1998). Cuanto más tiempo perdure la adherencia del paciente al tratamiento, mayor será el alejamiento de su anterior vida y más las oportunidades de mejoría. En segundo lugar, el propio consumo de drogas. La abstinencia de heroína, alcohol, cocaína y demás psicotropos continúa siendo condición necesaria para un buen resultado. En tercer lugar, la disminución de la delincuencia que se considera un referente fundamental. Finalmente, la ocupación en actividades no remuneradas, recreativas o la consecución de un empleo, que se valoran como muy positivas.

Han sido múltiples los estudios realizados sobre la bondad de estos procedimientos. Entre ellos, Vincent Dole y su escuela rechazan valorar el resultado que logra cada sujeto receptor de metadona en términos de mayor o menor adecuación a un registro de reglas y contingencias detalladas. Dole, en un estudio auspiciado por el National Institute of Drug Abuse (NIDA), preconiza más bien la evaluación basada en el rendimiento general del afectado más que en cumplimien- to estricto de normas prefijadas (Parrino, 1997, Fernández, 1999, Sánchez 2000; Fernández 2001).

La tendencia actual de evaluación de los TMM, sin abandonar los cuatro factores de la evaluación inicial, se completa valorando el estado de salud y otros factores asociados como la vía de administración, el riesgo de transmisión de infecciones, así como la percepción subjetiva del paciente sobre su estado de salud y su evolución (Brows, 1993; Ryan, 1996; Sánchez 2000). Para ello es necesario tener un diagnóstico lo más preciso posible y una valoración detallada de la situación de los pacientes en todos los planos expuestos, antes del acceso a un recurso de tratamiento. Sólo de ese modo se conseguirá evaluar los cambios registrados en los diferentes momentos del proceso en cada uno de los ámbitos y dimensiones sobre los que se espera que el TMM actúe.

Los objetivos del presente trabajo son: conocer la evolución de los pacientes en tratamiento con metadona en relación con la retención, la abstinencia, la situación laboral, los actos delictivos, y las variaciones en el nivel de gravedad medido. También se valoran los factores pronósticos para la retención.

\section{PACIENTESY MÉTODO}

Se presenta estudio longitudinal, prospectivo y multicéntrico. Se incluyó en la investigación una muestra de los pacientes drogodependientes que accedieron al TMM procedentes de las Unidades de Conductas Adictivas de Denia, Elche, La Vila Joiosa, Centro Penitenciario Cumplimiento de Alicante y del Centro Provincial de Drogodependencias de la Cruz Roja en Alicante. A todos se les informó de la investigación y otorgaron su consentimiento para el estudio.

La muestra estaba constituida por 197 pacientes que iniciaron de forma sucesiva tratamiento con metadona en las unidades referidas, entre junio y diciembre de 1998 con diagnóstico de dependencia a la heroína, según criterios de DSM-IV (DSM-IV 1995). No se incorporaron al estudio los pacientes que entraron por procedimiento de urgencia (embarazadas y pacientes hospitalizados) o derivados desde otros centros por traslado.

Se registraron las siguientes variables de identificación y socio-demográficas: edad, sexo, procedencia geográfica, nivel de instrucción, profesión, situación laboral. También se estudiaron los datos clínicos y toxicológicos que se citan: Infección por VIH, estadios CDC, marcadores clínicos y biológicos, tratamientos antirretrovirales, patología orgánica acompañante, consumos de sustancias, vía, tratamientos previos todos ellos recogidos en un protocolo clínico específico. 
TABLA 1. Distribución de Pacientes por Centros y Areas Sanitarias

\begin{tabular}{|l|l|l|l|l|l|l|l|}
\hline Area Sanitaria & $\begin{array}{l}\text { Unidad Conductas } \\
\text { Adictivas (UCAs) }\end{array}$ & $\begin{array}{l}\text { Número } \\
\text { Pacientes }\end{array}$ & $\begin{array}{l}\text { Número Total } \\
\text { entraron en TMM }\end{array}$ & Comarca & Población* & $\begin{array}{l}\text { Indicador de } \\
\text { actividad económica** }\end{array}$ & $\begin{array}{l}\text { N. Viviendas } \\
\text { nuevas }\end{array}$ \\
\hline Area 16-18 & Centro de Cruz Roja & 51 & 244 & Alacantí & 366.997 & 1.114 .308 & 501 \\
Area 12 & Denia & 36 & 58 & Marina Alta & 116.306 & 508.337 & 242 \\
Area 19 & Elx & 45 & 70 & Alt Vinalopó & 232.708 & 405144 & 883 \\
Area 15 & La Vila Joiosa & 28 & 40 & Marina Baixa & 117.431 & 449055 & 369 \\
Penitenciario & & 37 & 58 & & & & \\
\hline
\end{tabular}

* Censo de población de 1 de Enero de 1995 del Instituto Nacional de Estadística

** E. Eléctrica Miles /Kwh

Para la valoración del tratamiento con metadona se seleccionaron algunos indicadores clínicos y sociosanitarios: retención o permanencia del paciente en el TMM. Consumo de heroína, cocaína y otras sustancias, situación laboral, sobre la que se consideraron los meses de trabajo en los 12 meses anteriores y los meses trabajados durante el tratamiento; número de delitos previos y durante el tratamiento. Gravedad de la adicción obtenida mediante entrevista estandarizada descrita a continuación.

\section{Instrumentos de Medida}

El "Indicador del Tratamiento de Opiáceos" es la versión española de la entrevista clínica "Opiate Treatment Index" (OTI) de Darke y cols (1992) del National Drug and Alcohol Research Centre. University of New South Wales, Australia y validado en nuestro país por González Sáiz y el Grupo de Investigación en Medicina Social de la universidad de Cádiz. Valora siete dimensiones: Historia de tratamientos, Consumo de drogas, Conducta de Riesgo de Contagio de/ VIH, Funcionamiento Social, Actividad Delictiva, Estado de Salud y Ajuste Psicológico. Se consigue también una Puntuación Global sobre la gravedad de la adicción (González et al., 1997).

Para aportar al clínico una interpretación de las puntuaciones obtenidas en cada una de las escalas del OTI, éstas se han divido en quintiles. De esta forma, y para propósitos clínicos, la gravedad de los pacientes en cada área puede clasificarse como Alta, Media-Alta, Media, Media-Baja y Baja. Con ello, se permite la clasificación clínica de los grados de disfunción para cada una de las dimensiones de resultado del OTI. (González et al., 1997).

El análisis de los datos se realizó inicialmente describiendo las características socio-demográficas, clínicas, toxicológicas y de gravedad de la adicción. La comparación de medias de variables cuantitativas de más de dos grupos se realizó mediante el test ANOVA de un factor. El test de la t-Student para muestras relacionadas se utilizó para comparar medias de variables obtenidas al inicio y al año en TMM. El test de la $\chi^{2}$ se utilizó para contrastar dos variables cualitativas. Para estudiar las variables que influían en la retención se realizó un modelo de regresión logística. Se consideró la significación estadística para una $p<0,05$.

\section{RESULTADOS:}

Las características de la muestra se resumen en la Tabla 2. En cuanto al sexo 165 son hombres (84\%) y 32 son mujeres (16\%). La edad media es de 31,7 años con una desviación típica de 6,4.

\section{Resultados iniciales antes del tratamiento con metadona}

Entre los datos obtenidos destaca el bajo nivel cultural y la falta de profesión cualificada. Consumen múltiples sustancias además de la heroína: un $38.5 \%$ cocaína, 49\% cannabis, 35\% benzodiacepinas. Los diferentes perfiles de los pacientes correspondientes a sus centros de procedencia se comparan en la Tabla 2. La vía inicial en un $32 \%$ es la intravenosa. El uso en alguna ocasión de la vía intravenosa continúa estando presente en el $72.5 \%$. Siguen compartiendo material de inyección un $13 \%$ de la muestra. Los pacientes con consumo endovenoso presentan, por otro lado, medias de edad significativamente mayores que los que inhalan la sustancia $(p<0,001)$.

Los antecedentes judiciales están presentes en un $70 \%$.

Con respecto a la situación laboral destaca que sólo un 5.5\% presenta situación laboral estable y casi un $50 \%$ se encuentran en paro y de ellos sólo el $20 \%$ reciben algún tipo de prestación social (Tabla 2).

En cuanto a la autopercepción sobre el estado de salud en el momento de la entrevista y los 30 días anteriores, solo el $25 \%$ considera que está mal o muy mal. 
TABLA 2. Características Socio-Demográficas y clínicas en las diferentes Unidades y en el Penitenciario

\begin{tabular}{|c|c|c|c|c|c|c|c|c|}
\hline & $\begin{array}{l}\text { CPD } \\
\text { Alicante }\end{array}$ & $\begin{array}{l}\text { UCA } \\
\text { Denia }\end{array}$ & $\begin{array}{l}\text { UCA } \\
\text { Elche }\end{array}$ & $\begin{array}{l}\text { UCA } \\
\text { La Vila }\end{array}$ & $\begin{array}{l}\text { Penitenciario } \\
\text { Fontcalent }\end{array}$ & $p$ & $\begin{array}{l}\text { Numero de } \\
\text { pacientes }\end{array}$ & porcentaje \\
\hline $\begin{array}{l}\text { Edad media } \\
\text { Varones } \\
\text { Mujeres }\end{array}$ & $\begin{array}{l}31 \\
90 \\
10\end{array}$ & $\begin{array}{l}33 \\
64 \\
36\end{array}$ & $\begin{array}{l}29 \\
83 \\
17\end{array}$ & $\begin{array}{l}30 \\
82 \\
18\end{array}$ & $\begin{array}{l}28 \\
94 \\
6\end{array}$ & $\begin{array}{l}0.006 \\
0.004 \\
0.005\end{array}$ & $\begin{array}{l}165 \\
32\end{array}$ & $\begin{array}{l}83.5 \\
16.5\end{array}$ \\
\hline Convi familia & 66 & 72 & 72 & 46 & 5.4 & 0.009 & 109 & 54 \\
\hline Emigración & 52 & 62 & 35 & 50 & 40 & No sig* & & \\
\hline $\begin{array}{l}\text { Nivel de Instruc } \\
\text { Analfabetos } \\
\text { Primaria inc }\end{array}$ & $\begin{array}{l}8 \\
34 \\
\end{array}$ & 33 & $\begin{array}{l}6 \\
14 \\
\end{array}$ & $\begin{array}{l}4 \\
10 \\
\end{array}$ & $\begin{array}{l}4 \\
35\end{array}$ & $\begin{array}{l}0.03 \\
0,04\end{array}$ & $\begin{array}{l}10 \\
52\end{array}$ & $\begin{array}{l}5 \\
31\end{array}$ \\
\hline $\begin{array}{l}\text { Trabajo } \\
\text { Fijo } \\
\text { Eventual } \\
\text { Paro con sub } \\
\text { Paro sin sub }\end{array}$ & $\begin{array}{l}4 \\
25 \\
22 \\
41\end{array}$ & $\begin{array}{l}17 \\
16 \\
25 \\
22\end{array}$ & $\begin{array}{l}2 \\
12 \\
22 \\
47\end{array}$ & $\begin{array}{l}0 \\
32 \\
10 \\
42\end{array}$ & $\begin{array}{l}8 \\
12 \\
10 \\
13\end{array}$ & 0.000 & $\begin{array}{l}11 \\
34 \\
41 \\
69\end{array}$ & $\begin{array}{l}5,5 \\
17 \\
20 \\
34\end{array}$ \\
\hline $\begin{array}{l}\text { Tipo Trabajo } \\
\text { Hostelería } \\
\text { Construcción } \\
\text { Peones }\end{array}$ & $\begin{array}{l}8 \\
4 \\
2\end{array}$ & $\begin{array}{l}11 \\
11 \\
16\end{array}$ & $\begin{array}{l}8 \\
4 \\
6\end{array}$ & $\begin{array}{l}39 \\
7 \\
7\end{array}$ & $\begin{array}{l}5 \\
5 \\
8\end{array}$ & 0.000 & $\begin{array}{l}28 \\
11 \\
35\end{array}$ & \\
\hline Antece. Legales & 74 & 50 & 72 & 60 & 100 & 0.000 & 145 & 72.5 \\
\hline $\begin{array}{c}\text { Vía actual } \\
\text { IV } \\
\text { Otras }\end{array}$ & $\begin{array}{l}33 \\
67\end{array}$ & $\begin{array}{l}25 \\
75\end{array}$ & $\begin{array}{l}33 \\
67\end{array}$ & $\begin{array}{l}22 \\
78\end{array}$ & $\begin{array}{l}35 \\
65\end{array}$ & 0.000 & $\begin{array}{l}75 \\
121\end{array}$ & $\begin{array}{l}37 \\
59\end{array}$ \\
\hline Vía inicial & & & & & & 0.00 & & \\
\hline IV 43 & 63 & 52 & 28 & 40 & & 65 & 32 & \\
\hline Otras & 57 & 33 & 47 & 71 & 60 & & 132 & 65 \\
\hline IV alguna vez & 70 & 83 & 85 & 61 & 90 & 0.005 & 158 & 79 \\
\hline Ha Comp mater. Iny & 34 & 58 & 56 & 25 & 78 & 0.001 & 101 & 50 \\
\hline Comparte hoy\% & 6 & 11 & 14 & 4 & 32 & 0.001 & 27 & 13 \\
\hline $\begin{array}{c}\text { Drogo de. Familia } \\
\text { Heroína } \\
\text { Alcohol }\end{array}$ & $\begin{array}{l}30 \\
23 \\
4 \\
\end{array}$ & $\begin{array}{l}52 \\
39 \\
8 \\
\end{array}$ & $\begin{array}{l}52 \\
38 \\
4\end{array}$ & $\begin{array}{l}46 \\
25\end{array}$ & $\begin{array}{l}59 \\
48\end{array}$ & $\begin{array}{l}\text { No sig } \\
0.000 \\
0.000\end{array}$ & $\begin{array}{l}94 \\
68 \\
6 \\
\end{array}$ & $\begin{array}{l}47 \\
34 \\
3 \\
\end{array}$ \\
\hline VIH positivo & 18 & 36 & 18 & 18 & 62 & 0.000 & 59 & 29 \\
\hline Consumo Cocaína & 51 & 38 & 47 & 50 & 0 & 0.000 & 77 & 38.5 \\
\hline Consumo Cannabis & 16 & 30 & 54 & 42 & 51 & 0.000 & 78 & 49 \\
\hline Consumo benzodiacepinas & 27 & 28 & 46 & 32 & 43 & 0.002 & 71 & 35 \\
\hline Consumo Buprex & 0 & 22 & 2.1 & 4 & 0 & 0.000 & 19 & 5 \\
\hline Consumo de alcohol & 12 & 30 & 42 & 29 & 0 & 0.000 & 44 & 22 \\
\hline Ant. Tratamiento previo & 72 & 72 & 70 & 82 & 49 & 0.04 & 147 & 77 \\
\hline $\begin{array}{l}\text { Autopercepción de } \\
\text { Estado de Salud }\end{array}$ & & & & & & No sig & & \\
\hline $\begin{array}{l}\text { Bien o muy bien } \\
\text { Regular } \\
\text { Mal o muy mal }\end{array}$ & $\begin{array}{l}41 \\
37 \\
22\end{array}$ & $\begin{array}{l}22 \\
44 \\
34\end{array}$ & $\begin{array}{l}38 \\
31 \\
29\end{array}$ & $\begin{array}{l}30 \\
40 \\
30\end{array}$ & $\begin{array}{l}48 \\
40 \\
11\end{array}$ & & $\begin{array}{l}70 \\
73 \\
54\end{array}$ & $\begin{array}{l}35 \\
37 \\
25\end{array}$ \\
\hline $\begin{array}{l}\text { Autopercepción de } \\
\text { Estado de Salud } 30\end{array}$ & & & & & & 0.002 & & \\
\hline Bien o muy bien & 44 & 34 & 38 & 26 & 61 & & 78 & 41 \\
\hline Regular & 42 & 25 & 40 & 37 & 20 & & 75 & 38 \\
\hline Mal o muy mal & 13 & 42 & 21 & 37 & 19 & & 44 & 21 \\
\hline
\end{tabular}

Los datos estas referidos en porcentajes a excepción de la edad que es la media de la muestra.

*No sig.: No significativo.

\section{Evaluación al año de seguimiento}

Al mes se seguimiento, el $82 \%$ de los pacientes permanecieron en tratamiento, el $3.6 \%$ abandonaron y el resto fueron trasladados a otros centros o ingresaron en prisión. Al año la retención era del $57 \%$, el $16.3 \%$ abandonaron, el 3.9\% ingresaron en prisión, 2 pacientes fallecieron y el resto fueron trasladados a otros centros. Por centros, la retención fue del 77\% en Denia, 53\% en Vila Joiosa, 50\% en Elche y 33\% en CPD Alicante.

\section{El consumo de drogas}

El consumo de heroína durante los 11 meses de tratamiento llegó al $67 \%$. De éstos consumían a diario el $3 \%$. Menos de dos veces por semana el $14 \%$. Mas de dos veces por semana el 7\%. Entre 1-4 veces 
semana el 7\%. La dosis media estimada de heroína consumida es $246 \mathrm{mg}$. De ellos, utilizan la vía intravenosa el $45 \%$. La media de número de inyecciones es de 44.7 por año.

El 62\% consumió cocaína alguna vez durante los 11 meses. De éstos el $42 \%$ consumen por vía intravenosa y el $30 \%$ fumada en base. El 9\% consumió diariamente. La media de días de consumo es de 78 días en 11 meses. La distribución de la frecuencia de consumo fue: una vez al mes $41 \%$, entre 1 y 4 veces al mes $27 \%$, entre 1 y 2 veces por semana 9\%, más de dos veces por semana $11 \%$.

Comparte material de inyección el 28\%. La media de intercambios realizados fue del 0.84 (mínimo 0 , máximo 40). De los pacientes que en el control inicial compartían el material de inyección, solo 1 sigue compartiéndolo, sin embargo 7 pacientes que no compartían inicialmente durante el tratamiento en algún momento compartieron el material de inyección.

La prevalencia de infecciones en la muestra fue: Infección por el VIH 32\%. Los marcadores de anticuerpos de hepatitis $B$ fueron en un $45 \%$ positivos. Los anticuerpos de la Hepatitis $C$ estaban presentes en el $55 \%$.

Situación legal: se observa una disminución en los delitos, pasando del 30\% de la muestra que no había cometido delitos durante su tiempo de adicción a un $75 \%$ al año de seguimiento.

\section{Tabla 3. Puntuaciones en los Factores del OTI Intercentros inicial y al año de seguimiento}

\begin{tabular}{|c|c|c|c|c|c|c|c|}
\hline \multirow[b]{2}{*}{ Centro } & \multicolumn{3}{|c|}{ ANTES DEL PROGRAMA } & \multicolumn{3}{|c|}{1 AÑO DE PROGRAMA } & \multirow[b]{2}{*}{$p$} \\
\hline & $n$ & Media & DT & $n$ & Media & DT & \\
\hline \multicolumn{8}{|c|}{ OTI riesgo asociado a la infección por VIH } \\
\hline Alicante & 51 & 7,96 & 7,69 & 19 & 4,53 & 3,61 & \\
\hline Denia & 36 & 11,31 & 6,42 & 29 & 5,03 & 5,14 & \\
\hline Elche & 45 & 8,67 & 6,86 & 28 & 3,36 & 3,73 & \\
\hline La Vila & 28 & 5,89 & 6,51 & 18 & 4,39 & 4.73 & \\
\hline Fontcalent & 37 & 8,16 & 9,69 & 10 & 1,40 & 3.78 & \\
\hline Total & 197 & 8,48 & 7,65 & 104 & 4,03 & 4,38 & 0,001 \\
\hline \multicolumn{8}{|l|}{ OTI SOCIAL } \\
\hline Alicante & 51 & 19,14 & 6,92 & 19 & 19,21 & 11,47 & \\
\hline Denia & 36 & 19,36 & 7,86 & 29 & 16,90 & 9,17 & \\
\hline Elche & 45 & 21.40 & 5,35 & 26 & 15,77 & 5,15 & \\
\hline La Vila & 28 & 18,93 & 6,37 & 18 & 14,11 & 8,04 & \\
\hline Fontcalent & 37 & 24,19 & 6,65 & 10 & 15,00 & 6,43 & \\
\hline Total & 197 & 20,72 & 6,84 & 102 & 16,36 & 8,41 & 0.922 \\
\hline \multicolumn{8}{|l|}{ OTI ACTIVIDAD DELICTIVA } \\
\hline Alicante & 51 & 1,25 & 1,93 & 19 & 0.39 & 1,42 & \\
\hline Denia & 36 & 0,69 & 1,45 & 29 & 0,21 & 0,77 & \\
\hline Elche & 45 & 0,42 & 1,46 & 28 & 0,32 & 0,94 & \\
\hline La Vila & 28 & 1,07 & 2,04 & 18 & 0,00 & 0,00 & \\
\hline Fontcalent & 37 & 0,65 & 1,27 & 10 & 0,00 & 0,00 & \\
\hline Total & 197 & 0,82 & 1,66 & 103 & 0,21 & 0,87 & $0.347 \mathrm{NS}^{*}$ \\
\hline \multicolumn{8}{|l|}{ OTI SALUD } \\
\hline Alicante & 51 & 10,47 & 5,75 & 18 & 7,21 & 6.03 & \\
\hline Denia & 36 & 18,92 & 8,00 & 29 & 9,79 & 6,44 & \\
\hline Elche & 45 & 17,27 & 7,19 & 28 & 8,29 & 5,87 & \\
\hline La Vila & 28 & 12,50 & 7,25 & 18 & 6,56 & 5.46 & \\
\hline Fontcalent & 37 & 10,68 & 6,18 & 10 & 6,70 & 6,72 & \\
\hline Total & 197 & 13.89 & 7,63 & 104 & 8,06 & 6,09 & 0.000 \\
\hline \multicolumn{8}{|l|}{ OTI SALUD PSICOLOGICA QHG-28 } \\
\hline Alicante & 51 & 6,10 & 5,84 & 19 & 3.70 & 3,76 & \\
\hline Denia & 36 & 13,11 & 8,18 & 28 & 5,96 & 7,01 & \\
\hline Elche & 45 & 13,29 & 7,49 & 28 & 3,96 & 5.04 & \\
\hline La Vila & 28 & 11,14 & 7,76 & 18 & 3,38 & 5,86 & \\
\hline Fontcalent & 37 & 11,51 & 6,93 & 10 & 6,10 & 8,64 & \\
\hline Total & 197 & 10,81 & 7,62 & 103 & 4,58 & 5.98 & 0.109 NS* $^{*}$ \\
\hline \multicolumn{8}{|l|}{ PUNTUACION GLOBAL DEL OTI } \\
\hline Alicante & 51 & 47,98 & 16,75 & 18 & 35,00 & 20,00 & \\
\hline Denia & 36 & 66,72 & 20,90 & 28 & 38,23 & 19,50 & \\
\hline Elche & 45 & 64,96 & 19,24 & 26 & 32,50 & 16.65 & \\
\hline La Vila & 28 & 52,67 & 20,38 & 18 & 28,61 & 13.62 & \\
\hline Fontcalent & 37 & 58,07 & 23,28 & 10 & 29.20 & 20,27 & \\
\hline Total & 197 & 58,23 & 21,18 & 100 & 35,58 & 18.04 & 0.001 \\
\hline
\end{tabular}

${ }^{*}$ NS: No significativo. 
Tabla 4. Gravedad intercentro inicial antes de TMM y al año de seguimiento

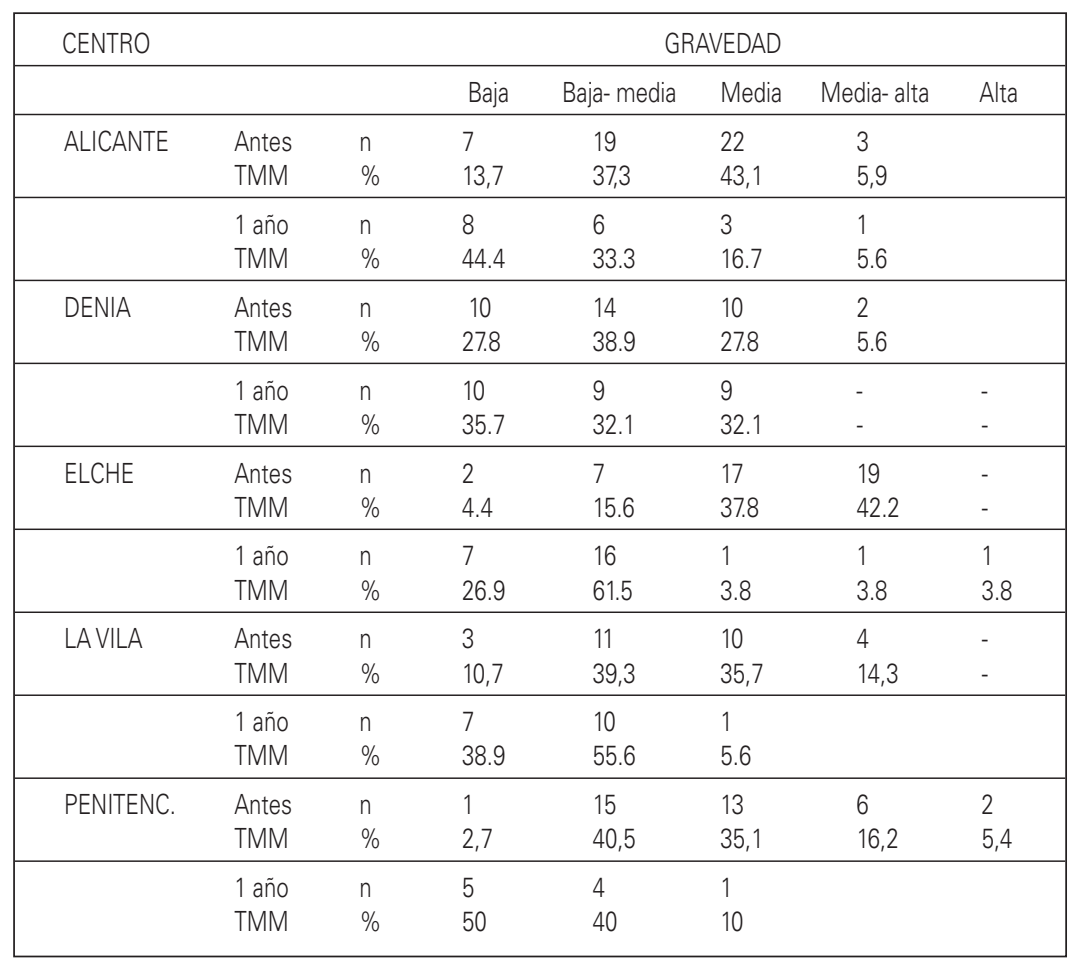

\section{TABLA 5. Riesgo de abandono del tratamiento con metadona}

\begin{tabular}{|lcccc|}
\hline & $B$ & $p$ & Exposición $\beta$ & IC $95 \%$ \\
\hline Antecedentes legales & 1,036 & 0,01 & 2,81 & $1,28-6,22$ \\
\hline Antecedentes Psiquiátricos & $-0,176$ & 0,69 & 0,838 & $0,35-2,04$ \\
\hline Dosis metadona & $-0,024$ & 0,08 & 0,97 & $0,95-1,00$ \\
\hline
\end{tabular}

Situación laboral: Se incrementan los pacientes que trabajan durante el seguimiento, desde un $49 \%$ antes del TMM hasta un $68 \%$ durante el año de seguimiento.

\section{Dosis de metadona.}

La dosis media de metadona al mes de tratamiento fue de $45 \mathrm{mg} /$ día (amplitud 10-75), y al año de tratamiento de 56 mg/día (amplitud 8-130).

\section{Gravedad de la adicción}

La puntuación de los factores del OTI al inicio y al año de tratamiento se puede ver en la Tabla 3. La mejoría de los resultados en las dimensiones del OTI al inicio y al final del tratamiento muestran significación estadística en todos los factores con excepción de las puntuaciones de delitos, social y del ajuste psicológico.

Las Puntuaciones Totales al inicio y al año de seguimiento se pueden observar en el diagrama de cajas representado en el Gráfico 1. Se observan diferencias significativas en los resultados de la muestra pareada al inicio y final del seguimiento. La media de la puntuación de la gravedad de la adicción de la muestra al inicio del tratamiento es de 58, con una desviación típica de 21, obteniéndose a los 12 meses una puntuación de 35 con desviación típica de 23.

Con respecto a las variaciones de los niveles de gravedad en la muestra pareada en el Gráfico 1 y Tabla 4 aparecen las modificaciones en la Puntuación Total y estratificada por niveles de gravedad.

La valoración que los pacientes hacen de los programas de tratamiento con metadona es entre buena y muy buena del $89 \%$, regular, mala y muy mala del $11 \%$.

\section{Abandonos}

El resultado del análisis de regresión logística para estudiar los abandonos se observa en la Tabla 5. La variable que mejor explicó las salidas del TMM, fue la que valora los antecedentes legales. Aquellos pacientes que habían cometido delitos mostraron un riesgo 2,8 veces superior de dejar el tratamiento que los que 


\section{GRÁFICO 1. Diferencias entre los valores de la mediana y los percentiles de la Puntuación total del OTI antesdel inicio $\mathrm{v}$ al año del tratamiento}

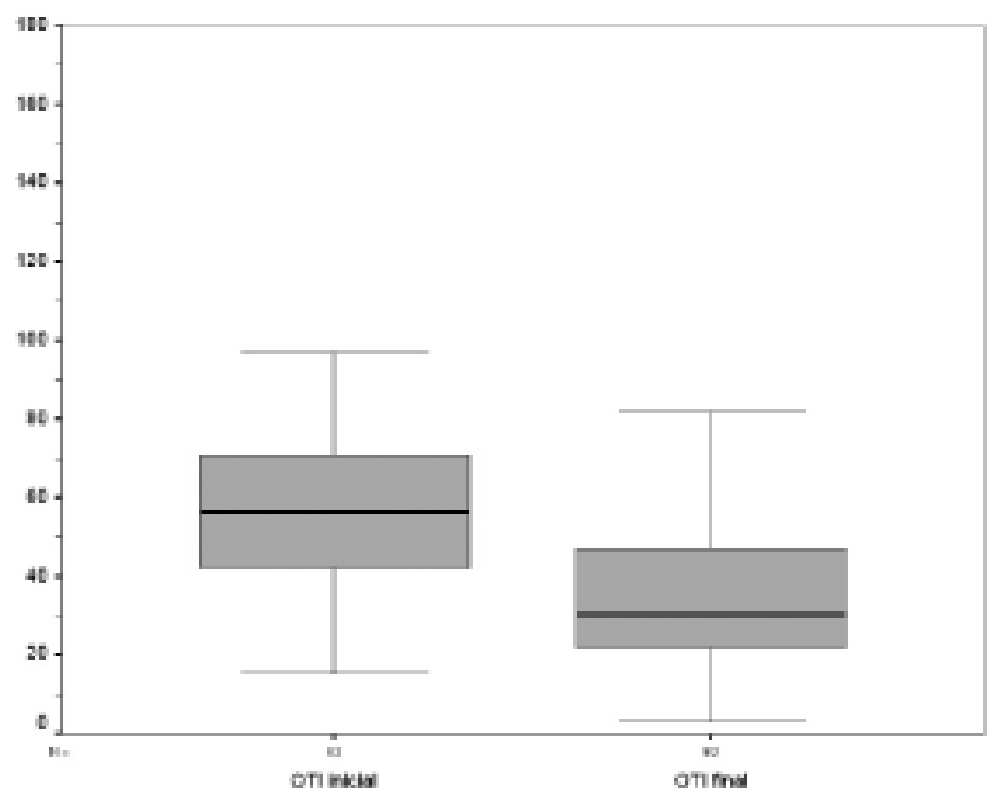

carecían de antecedentes. Ningún otro factor se relacionó con los abandonos.

No se observan diferencias significativas en las dosis de los pacientes que abandonan el tratamiento y los que se mantienen en seguimiento. Tampoco observamos diferencias con respecto a la retención al dicotomizar las dosis en mas de $60 \mathrm{mg} / \mathrm{día}$ y menos de $60 \mathrm{mg} / \mathrm{día}$.

También se valoraron los resultados de cada uno de los factores de del OTI con respecto a la retención por medio de un modelo de regresión logística, no obteniéndose significación estadística en ninguno de los factores.

\section{DISCUSIÓN}

La utilización de instrumentos de medición multifactoriales de la gravedad de la adicción, como el OTI, pretende estudiar los niveles de disfunción de cada uno de los aspectos que pueden influir en la mejora de la salud tanto en el plano individual como el de funcionamiento social de los pacientes dependientes.

A pesar del alto porcentaje de pacientes que han consumido en alguna ocasión heroína o cocaína durante el seguimiento sólo continúan realizando un consumo diario un $3 \%$ de heroína y $9 \%$ de cocaína, semejantes a los obtenidos por el Treatment Outcome Prospectyve Study (TOPS) (Fernández 1999b). Sin embargo las aportaciones de Marsch en un metanálisis realizado en 1998 sobre 11 artículos refiere que el
TMM se obtiene una disminución moderada del consumo de drogas ilegales. Con respecto a la vía de administración, nuestros usuarios han utilizando la vía intravenosa en alguna ocasión en un 70\%, y de manera predominante en un $37 \%$. En la muestra estudiada en Barcelona estos datos eran ligeramente superiores, con un $95 \%$ de vía endovenosa en alguna ocasión y un $48 \%$ de modo predominante (Torrens, 1997). Estas diferencias probablemente se deban a las variaciones en los hábitos de consumo detectados en las diferentes zonas del Estado. Ya que parece que existe un predominio del consumo intravenoso en las zonas norte de la península. Lo mismo sucede con la práctica de compartir el material de inyección, con un 13\% en nuestra muestra y un $21 \%$ en la de Barcelona (Torrens, 1997). En algunas muestras se encuentran que los pacientes en TMM comparten material de inyección en un $10 \%$ frente a un $27 \%$ en los pacientes que no están en tratamiento.

Se produce una mejoría significativa en el acceso al trabajo al año de seguimiento. Sin embargo no aparecen diferencias significativas en la escala de funcionamiento social del OTI inicial y final (Tabla 5). Esta escala mide la integración social no sólo a partir del empleo, si no también de la estabilidad residencial, los conflictos interpersonales y los apoyos sociales (González et al 1997). Nuestra muestra presenta un nivel de deterioro social muy intenso, difícilmente dependiente solamente de la consecución de trabajo. Este deterioro dificulta la reinserción y la nueva creación de redes o bien debido a la falta de recursos y programas de reinserción. 


\section{GRÁFICO 2. Cambios en los niveles de puntuación total del OTI antes del seguimiento del tratamiento}

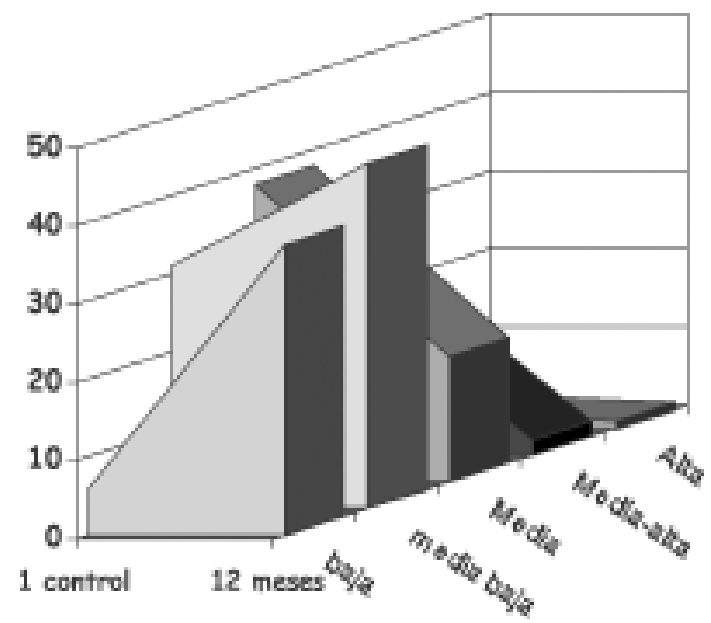

口baja $\square$ modiabaja DMedia E Modizalta $\square$ Alta

La dosis de media de metadona al mes de tratamiento es de 45 mg/día y al año de 56mg/día, estas dosis están por debajo de las recomendadas en la literatura y las encontradas en nuestro medio, dosis media a los 6 meses 58mg/día (rango 5-140, Iraugi et al 2002). Aunque por encima de las dosis medias utilizadas por estos recursos en años previos (Pellín, 2000). El Informe $N^{\circ} 1$ del Observatorio Español sobre Drogas señala que la dosis media global de metadona ponderada por el número de usuarios en cada centro fue de 71 mg/día en 1997. Sin embargo en algunos artículos publicados se reflejan dosis medias de metadona en torno a 40-50 mg. Strang et al. (1996) obtuvieron una dosis media para la metadona oral de 44,3mg. D'Ippoliti et al. (1998) calcularon que la dosis media fue $44 \mathrm{mg}$. En Estados Unidos, D'Aunno refleja una dosis media de $45 \mathrm{mg}$.

La utilización de la puntuación total de OTI permite obtener un nivel de gravedad de cada paciente y conocer si con el tratamiento se produce una disminución en las alteraciones sufridas por la dependencia. Nuestra muestra presenta una mejoría en la puntuación total del OTI, con mejoría de las dimensiones de conducta de riesgo con respecto a la infección por el VIH y la escala de salud. No mejoró la escala de actividad delictiva que mide los delitos cometidos en el último mes. La misma pregunta referida a los últimos 12 meses sí mostró una disminución desde el 70 \% de la muestra hasta 30 \%. Es posible que esta dimensión del OTI, al medir un espacio tiempo tan limitado, pierda información con respecto a la trayectoria de actividades ilegales. Sin embargo otros autores si hacen hincapié en la disminución de los delitos asociados al consumo de drogas ilegales (Marsch, 1998).
Al estratificar los resultados por categorías de gravedad observamos cómo al inicio del tratamiento el porcentaje de pacientes que se encuentran en las categorías de menor gravedad está alrededor del 45\% y cambia al año de seguimiento al $80 \%$. Existen diferencias entre los diferentes centros tanto en el porcentaje de acceso por sexos, como en el nivel de instrucción así como en las drogas consumidas y con respecto a las formas y vías de consumo. Los diferentes perfiles de cada centro vienen definidos por las características del área sanitaria y de los recursos humanos y medios de cada centro. Los centros situados al norte, Denia y La Vila Joiosa (Tabla 1) presentan situaciones económicas y de desarrollo social bien diferenciadas. Denia tiene un nivel de vida alto con casi pleno empleo y sin bolsas de marginación y tráfico en la zona. Sin embargo el centro inicia la dispensación de metadona con enfoques hacia la reducción de daños con lo que facilita el acceso a un perfil de paciente deteriorado con un nivel de gravedad entre media y alta del 70 \% con una media de edad mayor. Es un perfil que presenta poca patología social y gran peso de la comorbilidad psiquiátrica. En La Vila Joiosa existe un alto índice de emigración con un poblado de tráfico y muchos adictos viviendo alrededor de los traficantes, con graves problemas sociales y mayor nivel de desempleo. El perfil presenta mayor patología social. El centro está orientado hacia la reducción de daños, con gran parte de la población deteriorada ya captada en el recurso y con criterios flexibles. El centro de Alicante realiza la dispensación de toda la ciudad (Tabla 1) con rotaciones continuas del personal y el recurso esta masificado lo que obliga a horarios rígidos de dispensación en cuanto horario y accesibilidad del 
personal. El centro de Elche presenta un área de cobertura casi igual que Alicante ciudad, con empleo sumergido y con poblados de tráfico con graves problemas sociales. El perfil del usuario de este servicio presenta los niveles de gravedad más altos. El penitenciario presenta un nivel de deterioro social mayor. El uso de la vía intravenosa es mayor llegando al uso ocasional al 90\% de los adictos al inicio del tratamiento y el uso compartido del material de inyección se sitúa en el 32\%. En definitiva se observa, tras este análisis, la utilidad del OTI como instrumento global para estudiar no sólo perfiles individuales, si no también las características de los diversos centros y recursos de atención a los adictos. Se observa su capacidad para discriminar al grupo poblacional que atiende cada centro y el tipo de demanda que recibe. Las implicaciones para la asistencia y para el desarrollo de estrategias específicas basadas en la demanda son de enorme interés para profesionales y planificadores

En cuanto a la retención, uno de los pocos indicadores de mal pronóstico que encontramos en nuestra muestra son los antecedentes criminales. Ni los antecedentes psiquiátricos, ni la dosis, ni el consumo de cocaína, alcohol, cánnabis, ni la infección por VIH, significan mayor riesgo de abandonar el tratamiento. En la literatura las variables relativas al sujeto que ofrecen mayor consenso son la existencia de psicopatología previa y el consumo de cocaína, sin embargo algunos autores no encuentran variables pre tratamiento predictivas (Fernández 2001). Al clasificar la muestra en pacientes que reciben dosis inferiores o superiores a 60 mg/día, no encontramos diferencias en el porcentaje de abandonos. Estos hallazgos apoyan la tendencia de algunos grupos a individualizar las dosis a pesar de las recomendaciones generales sobre intervalos adecuados de dosis entre 80-120mg/día.

En definitiva, el nivel de gravedad de los pacientes dependientes a opiáceos disminuye de modo definido tras la entrada en TMM, mejoría que se mantiene al año de seguimiento. El no haber cometido delitos facilita la retención en tratamiento. Si consideramos, bajo el manto teórico del modelo de disminución de daños, que el objetivo de un programa de sustitución de opiáceos es disminuir la gravedad de la adicción y los riesgos asociados al consumo, podemos concluir que los TMM son eficaces, consiguen los objetivos propuestos y son un recurso valioso en el manejo de los pacientes con dependencia a opiáceos.

\section{BIBLIOGRAFÍA}

America Psychiatric Association. (1995) Practice guideline for the treatment of patients with subtances use disorders: alcohol, cocaine, opioids. American Journal of Psychiatry. 152 s11: 5-59,.
Insua P (1999) En. Programa de formación para la prevención de los problemas de salud asociados al consumo de drogas. Ed. Plan Nacional sobre Drogas.

Bell J; Zador D. (2000) A risk benefit analysis of methadone maintenance treatment. Drug Safety: 22, 179-190.

Baker, A. Heather, N. Wodak, A. y cols (1993) Evaluation of a cognitive- behavioural intervention for $\mathrm{VIH}$ prevention among injecting drug users. AIDS 7: 2, 247-56.

Baker, A. Kochan, N. Dixon, J. Y cols (1995). HIV risk-taking behaviour among injecting drug users currently, previously and never enrolled in methadone treatment.

Brown, LS; Siddiqui, NS. (1996) Natural history of HIV infection and predictors of survival in a cohort of HIV-1 seropositive inyecting drug users. J.Natl Med Assoc 88, 137-42.

D'Aunno T., Vaughn T.E., (1992) Variations in methadone treatment practices. Results from a national study. JAMA 267:253-258

De la Fuente L; Barrios G. (1996) Control de los problemas de salud asociados al consumo de drogas en España: Hacia una abordaje científico y priorizado. Gaceta Sanitaria 10 (57): 255-260.

D'Ippoliti D., Davoli M., Perucci C.A., Pasqualini F., Bargagli AM. (1998). Retention in treatment of heroin users in Italy: the role of treatment type and of methadone maintenance dosage. Drug Alcohol Depend. 52: 167171.

Darke, S. Hall, W. Wodak, A. and cols. (1992). Development and validation of a multidimensional instrument for assessing outcome of treatment among opioid users: The Opiate Treatment Index (OTI). Br. J. Addict 87: 583-602.

Darke, S. Kaye, S. Finlay- Jones, R. (1998). Drug use and injection risk-taking among prison methadone maintenence patients. Adicction 93: 8, 1169-1175.

Des Jarlais, D.C. Perlis, T. Friedman, S.R. y cols. (1998). Declining seroprevalence in very large HIV epidemic: inyecting drug users in New York City, 1991-1996. Am. J. Public Health. 88: 12 1801-1806.

Dole V.P, Nyswander M.E. (1965). A medical creatment for diacetyl morphine (heroin) addiction: A clinical trial with methadone hydrocloride. Journal of the American medical Association. 193: 80-84.

DSM-IV (1995) Manual diagnóstico y estadístico de los trastornos mentales. Ed Masson.

Duro P, Casas M, Colom J. (1994) Programas de objetivos intermedios para consumidores de opiáceos.

Effective medical treatment of opiate addiction. National Consensus Development Panel on Effective Medical Treatment of Opiate Addiction. (1999) JAMA 9: 280, 22, 1936-1943.

Eklund, C. Melin, L. Hiltunen, A. Borg, S. (1994). Detoxification from methadone maintenance treatment in Sweden: Long term outcome and effects on Quality of life and life situation. The international Journal of the addictions.

Fernández JJ; Marina PA.(1999) Manual de los tratamientos de mantenimiento con metadona. Oviedo. 
Fernández JJ ; González M.P.; Sáiz P.A. et al (1999b) Calidad de vida y severidad de la adicción en heroinómanos en mantenimiento prolongado con metadona. Adicciones 11 (1): 43-52.

Fernández Miranda JJ (2001) Efectividad de los programas de mantenimiento con metadona. Una revisión de los resultados de los estudios de evaluación. Med Clin (Barc) 116: 150-154.

Gaughwin, M. Solomon, P. Ali, R. (1998). Correlates of retention on the South Australian Methadone program 1981-

91. Aust N Z J Public Heatlth: 22,7, 771-776.

González Sáiz F.M. et al. (1997) Indicador del tratamiento de la adicción a opiáceos. Ed Servicio de publicaciones de la Universidad de Cádiz.

Gutiérrez E; Sáiz P.A.; García N. et al (2001) Evolución de la gravedad de la adicción a los dos años de tratamiento en pacientes heroinómanos. Adicciones 13, (1): 51-60

Hartel, D.M; Schoenbaum, E.E. (1998) Methadone treatment protecs against HIV infection: Two decades of experience in the Bronx, New York City. Public Health rep 113:1,107-115.

Informe $n^{\circ}$ 1. Observatorio Español sobre Drogas. (1998) Delegación del Gobierno para el Plan Nacional sobre Drogas. Secretaria General Técnica. Ed. Ministerio del Interior.

Iraugi I; Casas A; Celedonio MJ; García Serrano, Hormaza M. (2002) Estado de salud y calidad de vida en usuarios de un programa de mantenimiento con metadona. 3er Congreso Virtual de Psiquiatria. 1-28 febrero. http://www.psiquiatria.com/interpsiquis2002/5590.

Krekk M.J. (1983) Health consequences associated with the use of methadone. En Cooper J.R; Altman F; Brown $B, S$; Research on the treatment of narcotics addictions: State of the art. National Institute on Drug Abuse Research Monograph 83-1201. Rockville M.D: National Institute on Drug Abuse.

Langedam M.W; Van Haastrecht HJA; Coutinho RA; Van Ameijden EJC. (2001) The impact of Hard reduction based methadone treatment on mortality among heroin users. Am J public health :91, 774-780.
Lowinson J.H. and cols. (1997). Methadone Maintenance. Substance Abuse. A comprehensive textbook. Ed Willians and Wilkins.

Marsch L.A (1998) The efficacy of methadone maintenance interventions in reducing illicit opiate use, HIV risk, behavior and criminality: A meta-analysis. Addiction 93: 4, 515-532.

Mino, A. Casa, M. Gutiérrez, M. (1993) Sida y Toxicómanas Ed. Neurociencias. Barcelona

Murray, J. B. (1998) Effectiveness of methadone maintenance for heroin addiction. Psychol Rep 83: 1, 295-302.

O`Hare P. (1995).Introducción. La reducción de los daños relacionados con las drogas: Ed. Grup Igia

Pellin MC; Gimeno C; Barril J; Esteban J; Vilanova, E. (2000) Consumo legal de metadona en población drogodependiente de la provincia de Alicante. Rev. Toxicol. 17: 115-119.

Parrino M.W. (1997) Manual de tratamiento con metadona. Grup IGIA

Ruz, I. González Sáiz, F. Ruiz, F. (1998) Pacientes toxicómanos en programa de mantenimiento con metadona: evaluación del perfil de gravedad psicosocial y orgánico en atención primaria. Atención Primaria 21, 6, 384388.

Ryan, C.F. White, J.M. (1996) Health status at entry to methadone maintenance treatment using the SF-36 health survey questionarie. Addiction 91: 1, 39-45.

Sánchez Pardo L. (2000) Evaluación de la efectividad de los programas de sustitución opiáceos. Trastornos Adictivos $1: 2,56-72$.

Strang J., Sheridan J., Barber N. (1996). Prescribing injertable and oral methadone to opiate addicts: results from the 1995 national postal survey of community pharmacies in England and Wales. BMJ. 313: 270-272

Verster A; Buning E. (2000) Manual de metadona. EuroMethworK. Amsterdam.

Ward,J. Hall, W. Mattick, R.P. (1999) Role of maintenance treatment in opioide dependence. Lancet 16: 353, 9148, 221-226.

Weber, J.C. Kopferschmitt, J. (1998) Substitution therapy of drug addicts. Press med 12: 27, 39, 2088-99. 